\title{
Nitrile IR intensities characterize electric fields and hydrogen bond- ing in protic, aprotic, and protein environments
}

\author{
Jared Bryce Weaver, Jacek Kozuch, Jacob M. Kirsh, and Steven G. Boxer*
}

\author{
Supporting Information Placeholder
}

\begin{abstract}
Nitriles are widely used as vibrational probes; however, the interpretation of their IR frequencies is complicated by hydrogen bonding (H-bonding) in protic environments. We report a new vibrational Stark effect (VSE) that correlates the electric field projected on the $-\mathrm{C} \equiv \mathrm{N}$ bond to the transition dipole moment and, by extension, the nitrile peak area or integrated intensity. This linear VSE applies to both $\mathrm{H}$ bonding and non-H-bonding interactions. It can therefore be generally applied to determine electric fields in all environments. Additionally, it allows for semi-empirical extraction of the H-bonding contribution to the blueshift of the nitrile frequency. Nitriles were incorporated at $\mathrm{H}$-bonding and non-Hbonding protein sites using amber suppression, and each nitrile variant was structurally characterized at high resolution. We exploited the combined information now available from variations in frequency and integrated intensity and demonstrate that nitriles are a generally useful probe for electric fields.
\end{abstract}

Noncovalent interactions play pivotal roles in chemistry, from microscopic solvation environments to electrode interfaces, homo- and -heterogenous catalysts, biological membranes, and enzyme active sites. Despite their importance, it can be difficult to experimentally quantify the many types of interactions present in the condensed phase. One quantitative metric for these interactions is the electric field, often determined through the vibrational Stark effect (VSE) on the frequency. ${ }^{1}$

The first vibrational Stark spectra in an external applied electric field were analyzed for simple nitriles $(-\mathrm{C} \equiv \mathrm{N}) \cdot{ }^{2-4} \mathrm{Ni}-$ triles are often found on drugs, 5,6 and several methods have been developed to introduce nitrile probes at a range of positions in proteins, ${ }^{7-10}$ nucleic acids, ${ }^{11,12}$ biological membrane components, ${ }^{13}$ as well as in non-biological settings. ${ }^{14,15}$ Nitrile IR transitions are relatively strong and, unlike carbonyls $(-C=0)$, occur in an uncluttered region of the IR spectrum. Unfortunately, the interpretation of nitrile frequency shifts using the language of the VSE is complicated by a well-known blueshift in H-bonding solvents, ${ }^{10,16-18}$ undermining the utility of nitrile frequency shifts as direct quantitative probes of local electric fields, though changes in frequency can be useful at a qualitative level. ${ }^{19}$
The vibrational frequency of a well-isolated oscillator such as $-\mathrm{C} \equiv \mathrm{N}$ or $-\mathrm{C}=0$ can be approximated to first order in the field by the linear (dipolar) VSE:

$$
\bar{v}(\vec{F})=\bar{v}_{0}-\Delta \vec{\mu} \cdot \vec{F}
$$

where $\Delta \vec{\mu}$ is the vibrational Stark tuning rate or difference dipole moment which determines the sensitivity of a probe frequency to the electric field, $\vec{F}$, projected by the environment onto the probe's difference dipole moment (typically parallel to the bond axis), ${ }^{2}$ and $\bar{v}_{0}$ is the zero-field (vacuum) frequency. This frequency-field dependence can be calibrated by measuring vibrational solvatochromism and calculating the average fields using molecular dynamics (MD) simulations, as shown for a carbonyl group in Fig. 1A. This approach has been used with functionally relevant carbonyls to correlate electric fields at enzyme active sites with activation free energies. ${ }^{20-22}$ While a similar linear frequency-field correlation also applies to nitriles for aprotic solvents, the aforementioned blueshift in protic solvents lies far from this correlation, shown for benzonitrile's $-\mathrm{C} \equiv \mathrm{N}$ in Fig. 1B. Computational approaches which account for H-bonding frequency shifts are available but difficult to implement experimentally, ${ }^{16,23}$ and experiments characterizing $\mathrm{H}$-bonding 24,25 have thus far not been used to correct $\mathrm{H}$ bond frequency shifts in order to obtain electric fields.

While studying nitriles introduced as probes of electric fields in simple solvents and proteins, we observed substantial variation of the nitrile intensity, an effect noted before but not analyzed quantitatively. 15,26-28 Intriguingly, we found that the integrated intensity correlates with solvent field. As shown in the following, this new VSE circumvents the complication of the blueshift in H-bonding environments. The sensitivity of the transition dipole moment (TDM), $\vec{m}$, to an electric field can be expressed as

$$
\vec{m}(\vec{F})=\vec{m}_{0}-\vec{F} \cdot \underline{\mathrm{A}}-\vec{F} \cdot \underline{\underline{B}} \cdot \vec{F}+\vartheta\left(F^{3}\right)+\ldots
$$

where $\underline{\mathrm{A}}$ is the transition polarizability, $\underline{\mathrm{B}}$ is the transition hyperpolarizability, and $\vec{m}_{0}$ is the zero-field TDM. ${ }^{29}$ Paralleling Equation 1, $|\vec{m}|$ depends on $\underline{A}$ in a manner analogous to $\vec{v}$ 's dependence on $\Delta \vec{\mu}$. The $|\vec{m}|$ can be obtained directly from the concentration normalized peak area by

$$
|\vec{m}|=\sqrt{\frac{3 \epsilon_{0} h c \ln 10}{2 \pi^{2} N_{A}} \int \frac{\varepsilon(\bar{v})}{\bar{v}} d \bar{v}}
$$

where $\varepsilon(\bar{v})$ is the extinction coefficient and $\int \varepsilon(\bar{v}) d \bar{v}$ is the integrated peak area, and $\epsilon_{0}, h, c, N_{A}$, and $\bar{v}$ are the vacuum permittivity, Plank's constant, speed of light, Avogadro's number, and wavenumber frequency, respectively. ${ }^{30}$ 


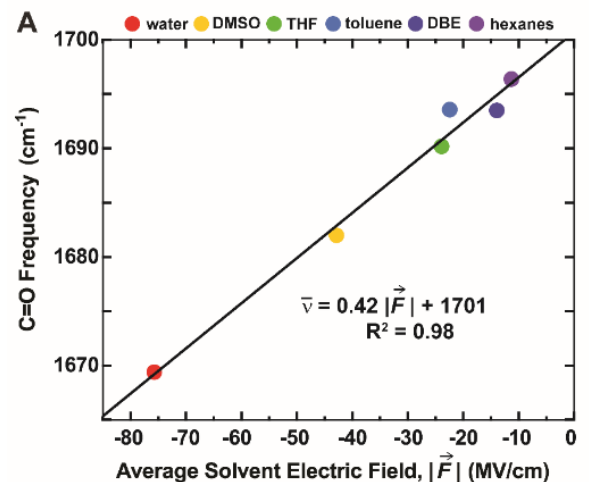

B

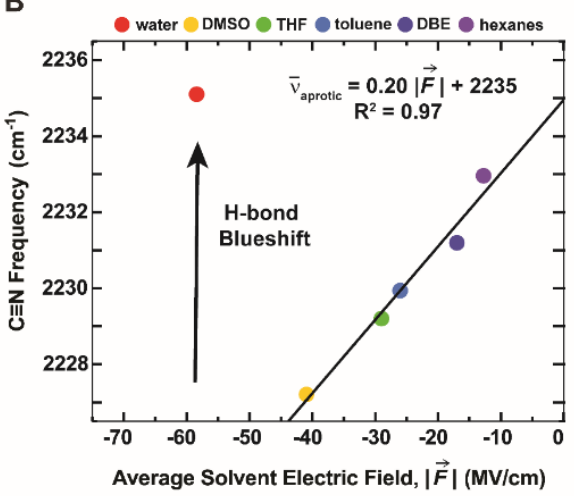

C

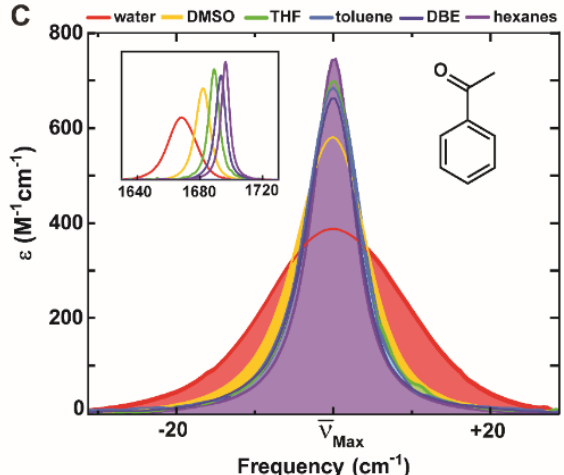

D

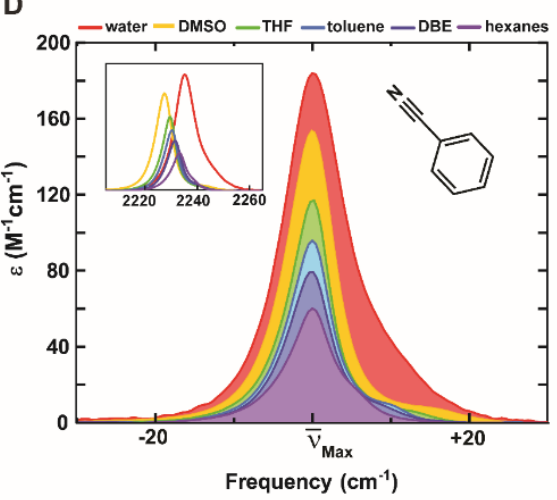

water DMSO THF toluene DBE hexanes

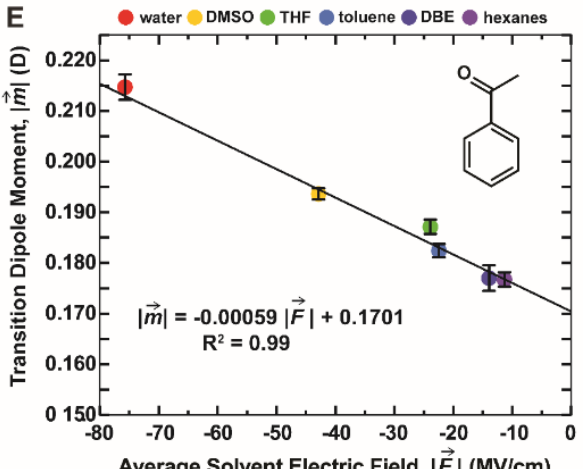

$F$

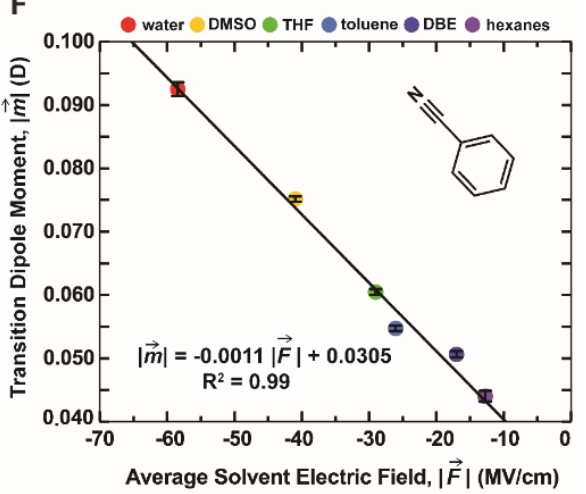

Figure 1. Peak frequency and peak area both change as a function of solvent electric field. (A, B) Plots of peak frequencies vs average solvent electric field calculated with polarizable MD for (A) ACP and (B) BZN. In (B) an arrow highlights the blueshift in -C $\equiv N$ frequency due to H-bonding in water. (C, D) Overlays of IR spectra of (C) ACP and (D) BZN where the IR peak position ( $\bar{v}_{\text {max }}$ ) has been centered in the plot and peaks are shaded to highlight peak area changes. Peak area increases with solvent polarity. Insets show noncentered spectra. (E, F) Plots of the TDM $v$ s the calculated average solvent electric field for both ACP (E) and BZN (F).

FTIR spectra were measured for acetophenone (ACP, $-\mathrm{C}=0$ band) and benzonitrile (BZN, $-\mathrm{C} \equiv \mathrm{N}$ band) in a variety of aprotic and protic solvents of increasing polarity (Figure 1C-D). The relative increase in peak area from hexanes to water is only $48 \%$ for ACP's - $\mathrm{C}=0$ but is $342 \%$ for BZN's $-\mathrm{C} \equiv \mathrm{N}$ (Figure $\mathrm{S} 4$ ). MD simulations using the polarizable AMOEBA09 force field 31 in Tinker 8.732 were used to calculate the average solvent electric field, $|\vec{F}|$, projected onto the $-\mathrm{C}=\mathrm{O}$ and $-\mathrm{C} \equiv \mathrm{N}$ probes in the same solvents, as described previously33-36. We demonstrate a linear trend when correlating $|\vec{m}|$, obtained from the peak areas using Equation 3, with the average $|\vec{F}|$ exerted on ACP's $\mathrm{C}=\mathrm{O}$ (Figure 1E). Importantly, for BZN's $-\mathrm{C} \equiv \mathrm{N}$, a linear relationship is also observed but with a steeper slope (Figure 1F), indicating a larger sensitivity of TDM to field relative to ACP's $-\mathrm{C}=0$ (see SI Section S6). In the context of the VSE on $|\vec{m}|$ (Eq. 2), the linear correlation between $|\vec{m}|$ and the average $|\vec{F}|$ demonstrates that higher order terms beyond $\underline{A}$ do not meaningfully contribute to tuning $|\vec{m}|$, as expected.3,37 Moreover, the linear relation between $|\vec{m}|$ and the average $|\vec{F}|$ is strictly monotonic for all solvents and well captured by the VSE, in contrast to the complicated picture for the $-\mathrm{C} \equiv \mathrm{N}$ frequency dependence observed in Fig 1B. 24,35 Taken together, by measuring the effect of solvent electric field on peak area, we are offered a direct, orthogonal calibration for measuring electric fields at nitriles which applies regardless of nitrile H-bonding status.

To demonstrate the utility of this new observable, we labeled photoactive yellow protein (PYP) with $o$-cyanophenylalanine (oCNF; Figure 2A-B) introduced site-specifically at multiple sites by amber suppression. ${ }^{38}$ PYP is a $14 \mathrm{kDa}$ protein with an endogenous chromophore which can be used to measure protein concentration. Four native phenylalanine sites were targeted for nitrile probe incorporation (Figures 2A and S11) such that the nitrile is placed in electrostatically distinct non-Hbonding and $\mathrm{H}$-bonding environments. High resolution X-ray structures $(<1.2 \AA \AA$; Figure $2 \mathrm{C}-\mathrm{F})$ show that the nitrile probes are in a single orientation and occupy distinct environments: F28oCNF possesses one H-bond donor ( $3.2 \AA$ heavy atom distance), F92oCNF has two donors (2.9 and $3.1 \AA$ ), and F62oCNF and F96oCNF are both in nonpolar environments (only carbons within $3.5 \AA$ Af the nitrile nitrogen).

The IR spectra of these PYP variants reveal significant variation of both the $-\mathrm{C} \equiv \mathrm{N}$ peak area and frequency (Figure $3 \mathrm{~A}$ ). A calibration curve was made for $o$-tolunitrile (oTN) as a model compound for oCNF (Figure S7C). The peak areas for the nitrile in F96oCNF and F62oCNF are similar to oTN's peak area in hexanes, while the areas for F28oCNF and F92oCNF are considerably larger and indicate water-like environments, consistent with expectations from X-ray structures. Translating the peak areas to TDMs gives values for $|\vec{m}|$ ranging from $0.050 \pm 0.002$ $\mathrm{D}$ in F96oCNF to $0.096 \pm 0.002 \mathrm{D}$ in F92oCNF (Table 1). Using the oTN TDM calibration (Figure S7C), we extract the average electric fields, which range from $-13 \pm 2 \mathrm{MV} / \mathrm{cm}$ to $-61 \pm 2$ $\mathrm{MV} / \mathrm{cm}$. Note that the electric field projected on the nitrile of F96oCNF is $\sim 4$ times smaller than that on F28oCNF's nitrile even though the peak frequencies are quite similar, illustrating that TDM tuning allows for identification of non-equivalent fields between species possessing nearly equivalent frequencies. 
A

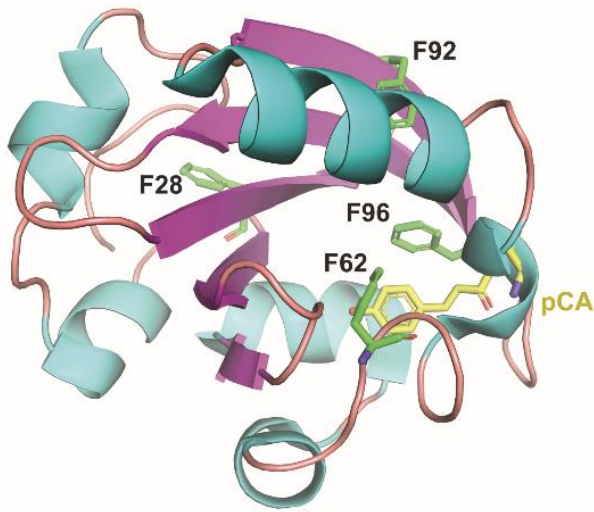

B
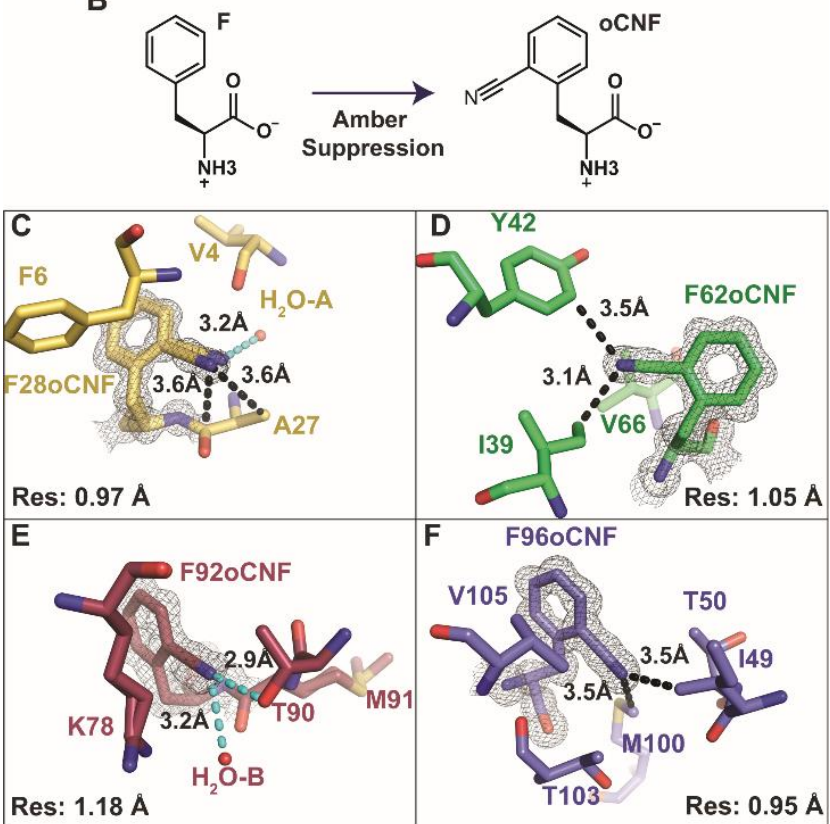

Figure 2. Overview of nitrile incorporation sites in PYP and their structural characterization. (A) The four phenylalanine sites targeted for nitrile probe incorporation are displayed in green, and the PYP chromophore, $p$-coumaric acid (pCA), is shown in yellow. (B) Native PYP phenylalanines (F) were replaced with genetically encoded $o$-cyanophenylalanine (oCNF) via amber stop codon suppression in four separate variants. Crystallographic structures of the nitrile site are displayed for F28oCNF (C), F62oCNF (D), F92oCNF (E), and F96oCNF (F) PYPs. Electron density maps for the oCNF in each structure are displayed $\left(2 \mathrm{mF}_{\mathrm{o}}-\mathrm{DF}_{\mathrm{c}}\right.$ contoured at $\left.1 \sigma\right)$ along with resolution of the crystal structure (bottom corner) and the distance between the nitrile nitrogen and the nearest two atoms (H-bond donor distances in cyan). For F28oCNF, two highly similar oCNF conformers are present; the distance of $\mathrm{H}_{2} \mathrm{O}-\mathrm{A}$ to the closer nitrile conformer is shown.

Various additive models have been developed to describe the behavior of the $-\mathrm{C} \equiv \mathrm{N}$ stretching frequency in the presence of H-bonds (electrostatic multipole expansion; ${ }^{23}$ separation into forces of various origin; 16 empirical models, ${ }^{18}$ see discussion in SI Section S17). We adopt a simple semi-empirical, experimentally useful model and partition the observed nitrile frequency $\left(\bar{v}_{\text {obs }}\right)$ relative to vacuum $\left(\bar{v}_{0}\right)$ into additive contributions from the shift due to the dipolar VSE $\left(\Delta \bar{v}_{\text {non-HB }}\right.$; obtained from solvatochromism in aprotic solvents) and from the frequency shift due to $\mathrm{H}$-bonding not captured by the dipolar VSE $\left(\Delta \bar{v}_{\mathrm{HB}}\right)$ :

$$
\bar{v}_{\mathrm{obs}}=\Delta \bar{v}_{\mathrm{HB}}+\Delta \bar{v}_{\text {non-HB }}+\bar{v}_{0}
$$

as illustrated in Figure 3C. Using the aprotic oCNF nitrile frequency-field calibration $\left(|\Delta \vec{\mu}|=0.19 \frac{\mathrm{cm}^{-1}}{\mathrm{MV} / \mathrm{cm}}, \bar{v}_{0}=2231.4 \mathrm{~cm}^{-1}\right.$; Figure S8B) and fields inferred from the TDM-field calibration (Figure 3B), $\Delta \bar{v}_{\text {non-Hв }}$ was determined (Figure 3C). Per Equation 4, $\Delta \bar{v}_{\mathrm{HB}}$ is the difference between $\bar{v}_{\mathrm{obs}}$ and $\Delta \bar{v}_{\text {non-HB }}+\bar{v}_{0}$ (Figure 3C). Using this approach, we observe a large range in $\Delta \bar{v}_{\mathrm{HB}}$, with the most H-bonding variants, F28oCNF and F92oCNF, possessing $\Delta \bar{v}_{\mathrm{HB}}$ of 7 and $21 \mathrm{~cm}^{-1}$, respectively (Table 1). This is an intriguing observation as $\Delta \bar{v}_{\mathrm{HB}}$ has been shown to be sensitive to $\mathrm{H}$-bond geometry (i.e., $\mathrm{H}$-bond angles and distances) ${ }^{23,39}$ and the geometry determines H-bond strength. 40

To further refine our analysis, we calculated the dependence of $\Delta \bar{v}_{\mathrm{HB}}$ on the heavy atom H-bond angle $(0 \cdots \mathrm{N} \equiv \mathrm{C}, \alpha$ in Figure 4 , proton positions are not resolved in the X-ray structures) for comparison with experimental results. Geometry optimization and vibrational frequency analysis [b3lyp/6-311++g(d,p)] were performed on oTN and a single water where $\alpha$ was varied from $70^{\circ}-175^{\circ}$ (see SI Section S16). H-bond distance was similar (3.0-3.2 ̊̊ over $90-175^{\circ}$ ) following geometry optimization, and DFT-calculated frequencies demonstrate an angle-dependence to the H-bond shift for oTN (Figure S19D) with the most significant blueshifts near $180^{\circ}$, similar to Choi et al. ${ }^{39}$ Given the $\mathrm{H}$-bond angles observed between $\mathrm{F} 92 \mathrm{oCNF}$ and T90 $\left(174^{\circ}\right)$ and between F28oCNF and $\mathrm{H}_{2} \mathrm{O}-\mathrm{A}\left(110^{\circ}\right)$, these DFT calculations predict $\Delta \bar{v}_{\mathrm{HB}}$ of $\sim 4$ and $\sim 22 \mathrm{~cm}^{-1}$ for F28oCNF and F92oCNF, respectively. While H-bond distance and number of donors ${ }^{16}$ also contribute to $\Delta \bar{v}_{\mathrm{HB}}$, we observe good agreement between predicted $\Delta \bar{v}_{\mathrm{HB}}$ and observed values $\left(7 \mathrm{~cm}^{-1}, 21 \mathrm{~cm}^{-1}\right)$. These results indicate the potential of $\Delta \bar{v}_{\mathrm{HB}}$ to report on $\mathrm{H}$-bonding geometries, though further benchmarking is necessary.

In summary, combined analysis of frequency and integrated peak intensity tuning with nitrile probes can be used to extract environmental electric fields and characterize H-bonding. TDM analysis enables quantification of significantly different fields for nitriles whose frequencies are nearly identical. We have utilized a simple phenomenological approach in this communication motivating a more comprehensive theoretical description of TDM and frequency tuning. It may be useful to re-evaluate intensity changes seen in other studies. ${ }^{15,27}$ Moreover, nitrile intensities can be used to determine electric fields and H-bonding in the extensive array of systems in which nitriles have been incorporated. $7,9-11,13,14,41$ 

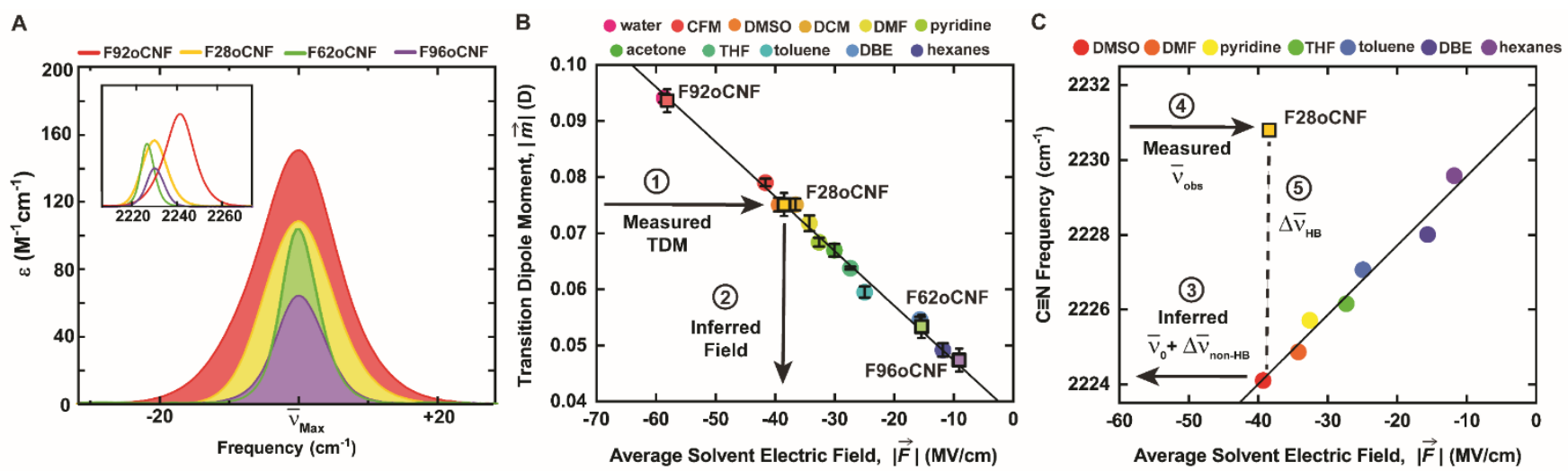

Figure 3. IR spectra of the $-\mathrm{C} \equiv \mathrm{N}$ stretch from incorporated oCNF residues at four sites in PYP (c.f. Figure 2) and extracted parameters. (A) Shaded IR overlay of the four labeled sites highlights the significant change in peak area from protein nitrile environments. Inset contains non-centered spectra. (B) Using the measured TDM (1), the electric field for proteins can be inferred from the oTN TDM $v s$ field calibration curve (2) (Figure S7C) as demonstrated for F28oCNF. (C) The aprotic solvent field $v s$ frequency calibration curve for oTN. Using the field inferred from the TDM (2), one can infer $\bar{v}_{0}+\Delta \bar{v}_{\text {non-HB }}(3)$, and by measuring $\bar{v}_{\text {obs }}(4)$, the blueshift $\left(\Delta \bar{v}_{\mathrm{HB}}\right)$ can be deduced (5)(Table 1).

Table 1. Extracted IR peak parameters for the oCNF nitrile incorporated at different PYP sites

\begin{tabular}{|c|c|c|c|c|c|c|}
\hline $\begin{array}{c}\text { Environ- } \\
\text { ment }\end{array}$ & $\begin{array}{c}\bar{v}_{\mathrm{obs}} \\
\left(\mathbf{c m}^{-1}\right)^{a}\end{array}$ & $\begin{array}{l}\text { FWHM } \\
\left(\mathrm{cm}^{-1}\right)^{a}\end{array}$ & $\begin{array}{l}|\overrightarrow{\boldsymbol{m}}| \\
\text { (D) }\end{array}$ & $\begin{array}{c}|\vec{F}| \\
(\mathrm{MV} / \mathrm{cm})\end{array}$ & $\begin{array}{c}\Delta \overline{\boldsymbol{v}}_{\text {non-HB }} \\
\left(\mathbf{c m}^{-1}\right)^{b}\end{array}$ & $\begin{array}{c}\Delta \bar{v}_{\mathrm{HB}} \\
\left(\mathrm{cm}^{-1}\right)^{b}\end{array}$ \\
\hline Hexanes & 2229.6 & 5.1 & $0.049 \pm 0.001$ & $-12 \pm 1$ & -2 & 0 \\
\hline Water & 2231.7 & 9.5 & $0.094 \pm 0.001$ & $-59 \pm 1$ & -11 & 11 \\
\hline F28oCNF & 2230.8 & 12.7 & $0.075 \pm 0.002$ & $-38 \pm 2$ & -6 & 7 \\
\hline F62oCNF & 2228.1 & 6.5 & $0.053 \pm 0.002$ & $-15 \pm 2$ & -3 & 0 \\
\hline F92oCNF & 2241.3 & 14.4 & $0.094 \pm 0.002$ & $-58 \pm 2$ & -11 & 21 \\
\hline F96oCNF & 2231.2 & 8.4 & $0.047 \pm 0.002$ & $-9 \pm 2$ & -2 & 2 \\
\hline
\end{tabular}

a Peak positions $\left(\bar{v}_{\text {obs }}\right)$ and FWHM all have an error of $<0.1 \mathrm{~cm}^{-1}$.

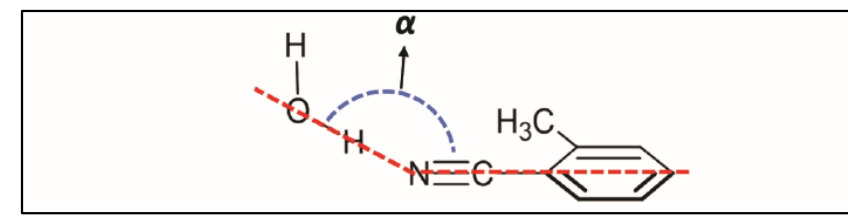

Figure 4. The heavy atom $\mathrm{H}$-bond angle $(\mathrm{O} \cdots \mathrm{N} \equiv \mathrm{C} ; \alpha)$ between a water and the nitrile of oTN, angle shown in blue.

\section{ASSOCIATED CONTENT}

\section{Supporting Information}

The Supporting Information is available free of charge on the ACS Publications website.

Experimental details on sample preparation and IR spectroscopy, IR spectra and TDM calibration curves for oTN, fixed-charge TDM calibration curves for ACP and BZN, Stark spectroscopy data and discussion, UV-vis spectra of PYP variants, mass spectra of PYP variants, crystallography procedure and structural details (PDF)

\section{AUTHOR INFORMATION}

\section{Corresponding Author}

Steven G. Boxer - Department of Chemistry, Stanford University, Stanford, California 94305-5012, United States; Email: sboxer@stanford.edu

\section{Authors}

Jared Bryce Weaver - Department of Chemistry, Stanford University, Stanford, California 94305-5012, United States;

Jacek Kozuch - Experimental Molecular Biophysics, Department of Physics, Freie Universität Berlin, 14195 Berlin, Germany;

Jacob M. Kirsh - Department of Chemistry, Stanford University, Stanford, California 94305-5012, United States;

Notes

The authors declare no competing financial interests.

\section{ACKNOWLEDGMENT}

J.B.W. was supported by a Stanford Center for Molecular Analysis and Design Fellowship. J.M.K. was supported by a Burt and Deedee McMurtry Stanford Graduate Fellowship. This work was supported by grants from the NSF (MCB-1915727) and NIH (R35GM118044) to S.G.B and the DFG (SFB1078 project B9) to J.K. We thank Dr. Chi-Yun Lin for helpful discussion regarding extraction of transition polarizabilities from vibrational Stark spectral fits.

\section{REFERENCES}


(1) Fried, S. D.; Boxer, S. G. Measuring Electric Fields and Noncovalent Interactions Using the Vibrational Stark Effect. Acc Chem Res, 2015, 48, 998-1006.

(2) Chattopadhyay, A.; Boxer, S. G. Vibrational Stark Effect Spectroscopy. J. Am. Chem. Soc. 1995, 117 (4), 1449-1450.

(3) Andrews, S. S.; Boxer, S. G. Vibrational Stark Effects of Nitriles I. Methods and Experimental Results. J. Phys. Chem. A 2000, 104 (51), 11853-11863.

(4) Andrews, S. S.; Boxer, S. G. Vibrational Stark Effects of Nitriles II. Physical Origins of Stark Effects from Experiment and Perturbation Models. J. Phys. Chem. A 2002, 106 (3), 469-477.

(5) Suydam, I. T.; Snow, C. D.; Pande, V. S.; Boxer, S. G. Electric Fields at the Active Site of an Enzyme: Direct Comparison of Experiment with Theory. Science 2006.

(6) Wang, X.; Wang, Y.; Li, X.; Yu, Z.; Song, C.; Du, Y. Nitrile-Containing Pharmaceuticals: Target, Mechanism of Action, and Their SAR Studies. RSC Medicinal Chemistry 2021, 12 (10), 1650-1671.

(7) Fafarman, A. T.; Webb, L. J.; Chuang, J. I.; Boxer, S. G. Site-Specific Conversion of Cysteine Thiols into Thiocyanate Creates an IR Probe for Electric Fields in Proteins. J. Am. Chem. Soc. 2006, 128 (41), 1335613357.

(8) Fafarman, A. T.; Sigala, P. A.; Schwans, J. P.; Fenn, T. D.; Herschlag, D.; Boxer, S. G. Quantitative, Directional Measurement of Electric Field Heterogeneity in the Active Site of Ketosteroid Isomerase. Proceedings of the National Academy of Sciences 2012, 109 (6), E299-E308.

(9) Adesina, A. S.; Świderek, K.; Luk, L. Y. P.; Moliner, V.; Allemann, R. K. Electric Field Measurements Reveal the Pivotal Role of CofactorSubstrate Interaction in Dihydrofolate Reductase Catalysis. ACS Catal. 2020, 10 (14), 7907-7914.

(10) Baiz, C. R.; Błasiak, B.; Bredenbeck, J.; Cho, M.; Choi, J.-H.; Corcelli, S. A.; Dijkstra, A. G.; Feng, C.-J.; Garrett-Roe, S.; Ge, N.-H.; Hanson-Heine, M. W. D.; Hirst, J. D.; Jansen, T. L. C.; Kwac, K.; Kubarych, K. J.; Londergan, C. H.; Maekawa, H.; Reppert, M.; Saito, S.; Roy, S.; Skinner, J. L.; Stock, G.; Straub, J. E.; Thielges, M. C.; Tominaga, K.; Tokmakoff, A.; Torii, H.; Wang, L.; Webb, L. J.; Zanni, M. T. Vibrational Spectroscopic Map, Vibrational Spectroscopy, and Intermolecular Interaction. Chem. Rev. 2020 120 (15), 7152-7218.

(11) Silverman, L. N.; Pitzer, M. E.; Ankomah, P. O.; Boxer, S. G.; Fenlon, E. E. Vibrational Stark Effect Probes for Nucleic Acids. J. Phys. Chem. B 2007, 111 (40), 11611-11613.

(12) Gai, X. S.; Fenlon, E. E.; Brewer, S. H. A Sensitive Multispectroscopic Probe for Nucleic Acids. J. Phys. Chem. B 2010, 114 (23), 7958-7966.

(13) Hu, W.; Webb, L. J. Direct Measurement of the Membrane Dipole Field in Bicelles Using Vibrational Stark Effect Spectroscopy. J. Phys. Chem. Lett. 2011, 2 (15), 1925-1930.

(14) Xiong, H.; Lee, J. K.; Zare, R. N.; Min, W. Strong Electric Field Observed at the Interface of Aqueous Microdroplets. J. Phys. Chem. Lett. 2020, 11 (17), 7423-7428.

(15) Staffa, J. K.; Lorenz, L.; Stolarski, M.; Murgida, D. H.; Zebger, I.; Utesch, T.; Kozuch, J.; Hildebrandt, P. Determination of the Local Electric Field at $\mathrm{Au} / \mathrm{SAM}$ Interfaces Using the Vibrational Stark Effect. $J$. Phys. Chem. C 2017, 121 (40), 22274-22285.

(16) Błasiak, B.; Ritchie, A. W.; Webb, L. J.; Cho, M. Vibrational Solvatochromism of Nitrile Infrared Probes: Beyond the Vibrational Stark Dipole Approach. Phys. Chem. Chem. Phys. 2016, 18 (27), 18094-18111.

(17) Reimers, J. R.; Hall, L. E. The Solvation of Acetonitrile. J. Am. Chem. Soc. 1999, 121 (15), 3730-3744.

(18) Aschaffenburg, D. J.; Moog, R. S. Probing Hydrogen Bonding Environments: Solvatochromic Effects on the CN Vibration of Benzonitrile. J. Phys. Chem. B 2009, 113 (38), 12736-12743.

(19) Kraskov, A.; von Sass, J.; Nguyen, A. D.; Hoang, T. O.; Buhrke, D.; Katz, S.; Michael, N.; Kozuch, J.; Zebger, I.; Siebert, F.; Scheerer, P.; Mroginski, M. A.; Budisa, N.; Hildebrandt, P. Local Electric Field Changes during the Photoconversion of the Bathy Phytochrome Agp2. Biochemistry 2021, 60 (40), 2967-2977.

(20) Fried, S. D.; Bagchi, S.; Boxer, S. G. Extreme Electric Fields Power Catalysis in the Active Site of Ketosteroid Isomerase. Science 2014, 346 (6216), 1510-1514.

(21) Schneider, S. H.; Kozuch, J.; Boxer, S. G. The Interplay of Electrostatics and Chemical Positioning in the Evolution of Antibiotic Resistance in TEM $\beta$-Lactamases; 2021; p 2021.05.27.446023.
(22) Zheng, C.; Mao, Y.; Kozuch, J.; Atsango, A.; Ji, Z.; Markland, T.; Boxer S. Electric Field Orientations in Solution and Enzyme Active Site Revealed by a Two-Directional Vibrational Probe. 2021.

(23) Lee, H.; Choi, J.-H.; Cho, M. Vibrational Solvatochromism and Electrochromism. II. Multipole Analysis. The Journal of Chemical Physics 2012, 137 (11), 114307

(24) First, J. T.; Slocum, J. D.; Webb, L. J. Quantifying the Effects of Hydrogen Bonding on Nitrile Frequencies in GFP: Beyond Solvent Exposure. J. Phys. Chem. B 2018, 122 (26), 6733-6743.

(25) Acharyya, A.; Mukherjee, D.; Gai, F. Assessing the Effect of Hofmeister Anions on the Hydrogen-Bonding Strength of Water via Nitrile Stretching Frequency Shift. J. Phys. Chem. B 2020, 124 (52), 1178311792.

(26) Eaton, G.; Pena-Nuñez, A. S.; Symons, M. C. R. Solvation of Cyanoalkanes [CH3CN and (CH3)3CCN]. An Infrared and Nuclear Magnetic Resonance Study. J. Chem. Soc., Faraday Trans. 1 1988, 84 (6), 2181-2193. (27) Delley, M. F.; Nichols, E. M.; Mayer, J. M. Interfacial Acid-Base Equilibria and Electric Fields Concurrently Probed by In Situ Surface-Enhanced Infrared Spectroscopy.J. Am. Chem. Soc. 2021, 143 (28), 1077810792.

(28) Yan, J.; Wilson, R. W.; Buck, J. T.; Grills, D. C.; Reinheimer, E. W.; Mani, T. IR Linewidth and Intensity Amplifications of Nitrile Vibrations Report Nuclear-Electronic Couplings and Associated Structural Heterogeneity in Radical Anions. Chem. Sci. 2021, 12 (36), 12107-12117.

(29) Bublitz, G. U.; Boxer, S. G. Stark Spectroscopy: Applications in Chemistry, Biology, and Materials Science. Annu. Rev. Phys. Chem. 1997 48 (1), 213-242.

(30) Park, E. S.; Boxer, S. G. Origins of the Sensitivity of Molecular Vibrations to Electric Fields: Carbonyl and Nitrosyl Stretches in Model Compounds and Proteins. J. Phys. Chem. B 2002, 106 (22), 5800-5806. (31) Ponder, J. W.; Wu, C.; Ren, P.; Pande, V. S.; Chodera, J. D.; Schnieders, M. J.; Haque, I.; Mobley, D. L.; Lambrecht, D. S.; DiStasio, R. A.; Head-Gordon, M.; Clark, G. N. I.; Johnson, M. E.; Head-Gordon, T. Current Status of the AMOEBA Polarizable Force Field. J. Phys. Chem. B 2010 114 (8), 2549-2564.

(32) Rackers, J. A.; Wang, Z.; Lu, C.; Laury, M. L.; Lagardère, L.; Schnieders, M. J.; Piquemal, J.-P.; Ren, P.; Ponder, J. W. Tinker 8: Software Tools for Molecular Design. J. Chem. Theory Comput. 2018, 14 (10), 52735289.

(33) Fried, S. D.; Bagchi, S.; Boxer, S. G. Measuring Electrostatic Field s in Both Hydrogen-Bonding and Non-Hydrogen-Bonding Environments Using Carbonyl Vibrational Probes. J. Am. Chem. Soc. 2013, 135 (30) 11181-11192.

(34) Schneider, S. H.; Boxer, S. G. Vibrational Stark Effects of Carbonyl Probes Applied to Reinterpret IR and Raman Data for Enzyme Inhibitors in Terms of Electric Fields at the Active Site. J. Phys. Chem. B 2016 $120(36), 9672-9684$.

(35) Deb, P.; Haldar, T.; Kashid, S. M.; Banerjee, S.; Chakrabarty, S.; Bagchi, S. Correlating Nitrile IR Frequencies to Local Electrostatics Quantifies Noncovalent Interactions of Peptides and Proteins. J Phys Chem B, 2016, 120, 4034-4046.

(36) Kozuch, J.; Schneider, S. H.; Zheng, C.; Ji, Z.; Bradshaw, R. T.; Boxer, S. G. Testing the Limitations of MD-Based Local Electric Fields Using the Vibrational Stark Effect in Solution: Penicillin G as a Test Case. J. Phys. Chem. B 2021, 13.

(37) Reimers, J. R.; Zeng, J.; Hush, N. S. Vibrational Stark Spectroscopy. 2. Application to the CN Stretch in HCN and Acetonitrile. J. Phys. Chem. 1996, 100 (5), 1498-1504.

(38) Tharp, J. M.; Wang, Y. S.; Lee, Y. J.; Yang, Y.; Liu, W. R. Genetic Incorporation of Seven Ortho-Substituted Phenylalanine Derivatives. ACS Chem. Biol., 2014, 9, 884-890.

(39) Choi, J.-H.; Oh, K.-I.; Lee, H.; Lee, C.; Cho, M. Nitrile and Thiocyanate IR Probes: Quantum Chemistry Calculation Studies and Multivariate Least-Square Fitting Analysis. J. Chem. Phys. 2008, 128 (13), 134506.

(40) Steiner, T. The Hydrogen Bond in the Solid State. Angewandte Chemie International Edition 2002, 41 (1), 48-76.

(41) Hopmann, K. H. Full Reaction Mechanism of Nitrile Hydratase: A Cyclic Intermediate and an Unexpected Disulfide Switch. Inorg. Chem. 2014, 53 (6), 2760-2762. 
For Table of Contents Only

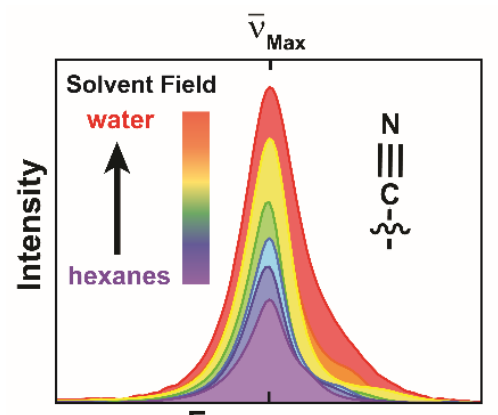

Frequency 